\title{
The influence of correlation on (de)localisation indices from a valence bond perspective
}

\author{
Guillaume Acke • Patrick Bultinck
}

Received: date / Accepted: date

\begin{abstract}
When going beyond the Hartree-Fock level to correlated methods one observes a significant reduction in the delocalisation index. This is commonly interpreted as a weakening of electron sharing due to electron correlation, although this is rather counter-intuitive to the concomitant energy lowering. In this study, we will use an analytical valence bond model and full CI calculations to show that this reduction in the delocalisation index actually goes hand in hand with increased covalent contributions at the expense of ionic contributions. This suggests that we should be careful in formulating interpretations of these results in (de)localisation indices.
\end{abstract}

Keywords valence bond theory · chemical bonding · delocalisation index

\section{Introduction}

Chemical bonding concepts play a pivotal role in explaining the increased stability of molecules compared to their corresponding assemblies of isolated atoms ${ }^{1}$. From a quantitative viewpoint, the energy lowering associated with moving from a so-called promolecule to a molecule has been scrutinised in great detail with respect to many facets such as the role of the kinetic energy and the corresponding electron density rearrangements ${ }^{2}$. From a qualitative viewpoint, an often used tool is that of the bond order ${ }^{3}$.

Historically, the bond order is defined as half the difference of the number of electrons in the bonding molecular orbitals and the number of electrons

Guillaume Acke

Krijgslaan 281 S3 B-9000 Belgium

Tel.: +3292644444

Patrick Bultinck

Krijgslaan 281 S3 B-9000 Belgium

Tel.: +3292644423

E-mail: Patrick.Bultinck@UGent.be 
in the antibonding orbitals involved in describing a given bond. As such, in the simplest case of $\mathrm{H}_{2}$ at the Hartree-Fock level with a minimal basis set, the bond order is one. Despite inherently not being a quantum chemical observable, the bond order concept has been extended to a wide class of bond indices ${ }^{4}$, including but not limited to (de)localisation indices ${ }^{5-7}$ - sometimes called shared electron distribution indices ${ }^{8}-$ and bond orders ${ }^{9-11}$.

The (de)localisation indices can be derived ${ }^{12}$ from the Domain-Averaged Fermi Hole or DAFH ${ }^{8,13-16}$ associated with an atomic domain $\Omega_{A}$ in position space. Note that this is not the only approach to derive (de)localisation indices. Alternatively, one can introduce (de)localisation indices as (co)variances ${ }^{17-19}$. In this work, however, we follow the density based approach to examine the effect of electron correlation on (de)localisation indices. The Domain-Averaged Fermi Hole $g\left(\boldsymbol{r}_{a} ; \Omega_{A}\right)$ is given by

$$
g\left(\boldsymbol{r}_{a} ; \Omega_{A}\right)=\rho^{(1)}\left(\boldsymbol{r}_{a}\right) \int_{\Omega_{A}} \rho^{(1)}\left(\boldsymbol{r}_{b}\right) \mathrm{d} \boldsymbol{r}_{b}-\int_{\Omega_{A}} \rho^{(2)}\left(\boldsymbol{r}_{a}, \boldsymbol{r}_{b}\right) \mathrm{d} \boldsymbol{r}_{b},
$$

where $\rho^{(1)}\left(\boldsymbol{r}_{a}\right)$ is the spin-integrated first-order reduced density

$$
\rho^{(1)}\left(\boldsymbol{r}_{a}\right)=\left\langle\Psi\left|\sum_{i=1}^{N} \delta\left(\boldsymbol{r}_{i}-\boldsymbol{r}_{a}\right)\right| \Psi\right\rangle
$$

and $\rho^{(2)}\left(\boldsymbol{r}_{a}, \boldsymbol{r}_{b}\right)$ is the spin-integrated second-order reduced density

$$
\rho^{(2)}\left(\boldsymbol{r}_{a}, \boldsymbol{r}_{b}\right)=\left\langle\Psi\left|\sum_{i \neq j}^{N} \delta\left(\boldsymbol{r}_{i}-\boldsymbol{r}_{a}\right) \delta\left(\boldsymbol{r}_{j}-\boldsymbol{r}_{b}\right)\right| \Psi\right\rangle
$$

Similar to the first-order reduced density, the DAFH can be represented as a matrix in an orbital basis ${ }^{12}$. The (localised) eigenvectors and eigenvalues of this matrix have been shown to give insight into diverse bonding patterns ${ }^{20-27}$. The localisation index $\Delta\left(\Omega_{A}, \Omega_{A}\right)$ is obtained by performing an additional integration of $g\left(\boldsymbol{r}_{a} ; \Omega_{A}\right)$ over the domain $\Omega_{A}$

$$
\begin{aligned}
\Delta\left(\Omega_{A}, \Omega_{A}\right) & =\int_{\Omega_{A}} g\left(\boldsymbol{r}_{a} ; \Omega_{A}\right) \mathrm{d} \boldsymbol{r}_{a} \\
& =\int_{\Omega_{A}} \rho^{(1)}\left(\boldsymbol{r}_{a}\right) \int_{\Omega_{A}} \rho^{(1)}\left(\boldsymbol{r}_{b}\right) \mathrm{d} \boldsymbol{r}_{b}-\int_{\Omega_{A}} \int_{\Omega_{A}} \rho^{(2)}\left(\boldsymbol{r}_{a}, \boldsymbol{r}_{b}\right) \mathrm{d} \boldsymbol{r}_{b} \mathrm{~d} \boldsymbol{r}_{a},
\end{aligned}
$$

and the delocalisation index $\Delta\left(\Omega_{A}, \Omega_{B}\right)$ is obtained by performing an additional integration of $g\left(\boldsymbol{r}_{a} ; \Omega_{A}\right)$ over a domain $\Omega_{B}$, different from $\Omega_{A}$

$$
\Delta\left(\Omega_{A}, \Omega_{B}\right)=2\left[\int_{\Omega_{B}} \rho^{(1)}\left(\boldsymbol{r}_{a}\right) \int_{\Omega_{A}} \rho^{(1)}\left(\boldsymbol{r}_{b}\right) \mathrm{d} \boldsymbol{r}_{b}-\iint_{\Omega_{B}} \int_{\Omega_{A}} \rho^{(2)}\left(\boldsymbol{r}_{a}, \boldsymbol{r}_{b}\right) \mathrm{d} \boldsymbol{r}_{b} \mathrm{~d} \boldsymbol{r}_{a}\right] .
$$


At the Hartree-Fock level of theory, these indices lead to chemically very appealing results. For instance, for $\mathrm{H}_{2}$, the delocalisation index is close to one for a variety of domain partitionings. Likewise, for other diatomics there is a remarkable resemblance between classical bond orders and the delocalisation index (for example, $\mathrm{N}_{2}$ has a delocalisation index of nearly 3 ). This success in the sense of giving data that match expectations has resulted in varied usage to detect chemical bonding in many molecules, including the detection of interactions that would otherwise not have attracted much attention. For instance, delocalisation indices and multicenter indices have been used to reveal intricate bonding patterns underlying aromaticity ${ }^{28-30}$.

When going beyond the Hartree-Fock level of theory towards correlated methods, the chemical interpretation of these (de)localisation indices becomes less clear-cut ${ }^{31}$. Indeed, accounting for electron correlation leads to a significant reduction in the delocalisation index ${ }^{7,32}$. This gives rise to statements such as "the inclusion of Coulomb correlation induces a decrease in electron sharing ...because, on average, electrons repel more strongly than if only same spin correlation is included" ${ }^{33}$, implying that the resulting decrease in electron sharing is beneficial to lowering the energy. In this study, we will revisit the analytic models proposed by Ponec et al. ${ }^{34}$ and full configuration interaction wave functions projected on valence bond structures to add more insight based on a valence bond type description as this introduces covalency and ionicity in a natural way.

Before proceeding with the discussion of this model, we first make some important comments. First, the frequently used probabilistic interpretation of these delocalisation indices can be problematic. In this interpretation, one considers the diagonals of the second order density matrix as related to a probability to find two interacting electrons in infinitesimal volume elements and the product of two first order densities as related to the probabilities associated with two non-interacting electrons. If this were the case, the trace or equivalently the integral over all space for both electrons of the difference of these probabilities should be zero (i.e. $\Delta\left(\mathbb{R}^{3}, \mathbb{R}^{3}\right)=0$ ) and not N. This was known to Bader ${ }^{5}$ and gave rise to the properly probability-based hole on the one hand and the density-based hole studied here ${ }^{5}$. As such, probabilistic interpretations of density-based indices should be reconsidered.

Second, the chemically appealing delocalisation index of one for $\mathrm{H}_{2}$ at the Hartree-Fock level, is rather problematic in itself. Fermi correlation originates from the interaction between two like-spin electrons, of which there are none in the ground state for $\mathrm{H}_{2}$. As stated clearly by Kutzelnigg ${ }^{35}$, exchange has a contribution with trace $-N$ (the number of electrons) to the pair density due to self-pairing added to the direct and the exchange part. It is this self-pairing, and not genuine exchange, that gives rise to the trace $N$.

A third problem is that the "chemical bond", like many commonly accepted chemical concepts, has no unique definition ${ }^{36}$. In the remainder of this study, we opt to consider covalency as "VB covalency", meaning the equal electron sharing part of the valence bond wave function discussed below. 


\section{Result and discussion}

Let us first assume that one can extract bond indices for the $\mathrm{H}_{2}$ molecule using spin-free delocalisation indices, ignoring the fact that there is no strict Fermi correlation possible in singlet $\mathrm{H}_{2}$ nor Coulomb correlation at the Hartree-Fock level. We merely use the position space expressions for (de)localisation indices without reconsidering how these result from the singlet, spin-dependent parent wave function.

Let us introduce a minimal basis set with atom centered, in general nonorthogonal, normalised spatial atomic orbitals $\phi_{1}$ and $\phi_{2}$ with $\left\langle\phi_{1} \mid \phi_{2}\right\rangle=s$. We next introduce the Hartree-Fock molecular spatial orbitals

$$
\begin{aligned}
& \psi_{1}=\frac{1}{\sqrt{2+2 s}}\left(\phi_{1}+\phi_{2}\right) \\
& \psi_{2}=\frac{1}{\sqrt{2-2 s}}\left(\phi_{1}-\phi_{2}\right) .
\end{aligned}
$$

The molecular Hartree-Fock wave function for the ground state $\left(\Psi_{H F}\right)$ corresponds to the double occupation of $\psi_{1}$, once with an electron with $\alpha$ spin and once for an electron with $\beta$ spin coupled to a singlet eigenfunction of $\hat{S}^{2}$ and $\hat{S}_{z}$. In the full configuration interaction (CI) wave function, $\Psi_{H F}$ is combined with the doubly excited determinant $\Psi_{11}^{22}$ where $\psi_{2}$ is doubly occupied. In an alternative approach, a valence bond picture can be used where a covalent and ionic wave function is found as

$$
\begin{gathered}
\Phi_{\text {cov }}\left(\boldsymbol{x}_{1}, \boldsymbol{x}_{2}\right)=\frac{1}{\sqrt{2+2 s^{2}}}\left[\phi_{1}\left(\boldsymbol{r}_{1}\right) \phi_{2}\left(\boldsymbol{r}_{2}\right)+\phi_{2}\left(\boldsymbol{r}_{1}\right) \phi_{1}\left(\boldsymbol{r}_{2}\right)\right] \Theta\left(\sigma_{1}, \sigma_{2}\right) \\
\Phi_{\text {ion }}\left(\boldsymbol{x}_{1}, \boldsymbol{x}_{2}\right)=\frac{1}{\sqrt{2+2 s^{2}}}\left[\phi_{1}\left(\boldsymbol{r}_{1}\right) \phi_{1}\left(\boldsymbol{r}_{2}\right)+\phi_{2}\left(\boldsymbol{r}_{1}\right) \phi_{2}\left(\boldsymbol{r}_{2}\right)\right] \Theta\left(\sigma_{1}, \sigma_{2}\right),
\end{gathered}
$$

with

$$
\Theta\left(\sigma_{1}, \sigma_{2}\right)=\frac{\alpha\left(\sigma_{1}\right) \beta\left(\sigma_{2}\right)-\beta\left(\sigma_{1}\right) \alpha\left(\sigma_{2}\right)}{\sqrt{2}} .
$$

The Hartree-Fock and CI wave functions can be expressed in terms of these valence bond structure wave functions by projection using the projection operators:

$$
\begin{gathered}
\left|\Phi_{\text {cov }}\right\rangle \Delta_{\text {cov }, \text { cov }}^{-1}\left\langle\Phi_{\text {cov }}|+| \Phi_{\text {cov }}\right\rangle \Delta_{\text {cov }, \text { ion }}^{-1}\left\langle\Phi_{\text {ion }}\right| \\
\left|\Phi_{\text {ion }}\right\rangle \Delta_{\text {ion,cov }}^{-1}\left\langle\Phi_{\text {ion }}|+| \Phi_{\text {ion }}\right\rangle \Delta_{\text {ion,ion }}^{-1}\left\langle\Phi_{\text {cov }}\right|
\end{gathered}
$$

$\Delta^{-1}$ is the inverse of the metric matrix with e.g. $\Delta_{\text {cov,ion }}=\left\langle\Phi_{\text {cov }} \mid \Phi_{\text {ion }}\right\rangle$. It is immediately clear that the Hartree-Fock wave function corresponds to a mixture of covalent and ionic valence bond functions with equal coefficients. Therefore, this wave function attaches equal importance to the ionic and the 
covalent parts. As is well-known, this is obviously a rather poor wave function to describe the molecular dissociation curve.

In order to show the effect of electron correlation on the delocalisation indices and on the nature of these wave functions in this simple molecule, we will use the simple analytic model of Ponec et al. ${ }^{34}$. The dissociation of the bonding electron pair in a homonuclear diatomic molecule is described by the following wave function

$$
\Psi_{\mathrm{VB}}\left(\boldsymbol{x}_{1}, \boldsymbol{x}_{2}\right)=\cos (\omega) \Psi_{\mathrm{cov}}\left(\boldsymbol{x}_{1}, \boldsymbol{x}_{2}\right)+\sin (\omega) \Psi_{\mathrm{ion}}\left(\boldsymbol{x}_{1}, \boldsymbol{x}_{2}\right)
$$

With this model, we can describe several chemically relevant situations:

- The $\omega=\frac{\pi}{4}$ case corresponds to a Hartree-Fock description, giving equal weights to covalent and ionic contributions, and can be taken as a model for the Hartree-Fock ${ }^{1} \Sigma_{g}^{+}$ground state of $\mathrm{H}_{2}$.

- The $\omega=0$ case corresponds to a Heitler-London ground state description with only covalent contributions, and can be taken as a model for the ${ }^{1} \Sigma_{g}^{+}$ ground state of $\mathrm{H}_{2}$ at the limit of infinite internuclear distance.

- The $\omega=\frac{\pi}{2}$ case corresponds to a Heitler-London description with only ionic contributions, and can be taken as a model for the $\mathrm{S} 2{ }^{1}{ }^{1} \Sigma_{g}^{+}$excited state of $\mathrm{H}_{2}$.

Within this model, the orbitals are confined to their domains, with orbital $a$ confined to domain $\Omega_{A}$ and similarly, orbital $b$ confined to domain $\Omega_{B}$

$$
\begin{gathered}
\int_{\Omega_{A}} a(\boldsymbol{r}) a(\boldsymbol{r}) \mathrm{d} \boldsymbol{r}=1 \\
\int_{\Omega_{A}} b(\boldsymbol{r}) b(\boldsymbol{r}) \mathrm{d} \boldsymbol{r}=0 \\
\int_{\Omega_{A}} a(\boldsymbol{r}) b(\boldsymbol{r}) \mathrm{d} \boldsymbol{r}=0 .
\end{gathered}
$$

Ponec et al. ${ }^{34}$ derived that for this analytical model the DAFH $g\left(\boldsymbol{r}_{a} ; \Omega_{A}\right)$ is given by

$$
g\left(\boldsymbol{r}_{a} ; \Omega_{A}\right)=\cos ^{2}(\omega) a\left(\boldsymbol{r}_{a}\right)^{2}+2 \cos (\omega) \sin (\omega) a\left(\boldsymbol{r}_{a}\right) b\left(\boldsymbol{r}_{a}\right)+\sin ^{2}(\omega) b\left(\boldsymbol{r}_{a}\right)^{2} .
$$

As such, the localization and delocalisation indices for this model are given by

$$
\begin{aligned}
& \Delta\left(\Omega_{A}, \Omega_{A}\right)=\cos ^{2}(\omega) \\
& \Delta\left(\Omega_{A}, \Omega_{B}\right)=2 \sin ^{2}(\omega),
\end{aligned}
$$

as plotted in figure 1 .

In agreement with the results reported by Pendás and Francisco ${ }^{37}$, for a Hartree-Fock type wave function $\left(\omega=\frac{\pi}{4}\right)$ the localization index is equal to $\frac{1}{2}$ and the delocalization index to 1 . At the dissociation limit of the ground state 


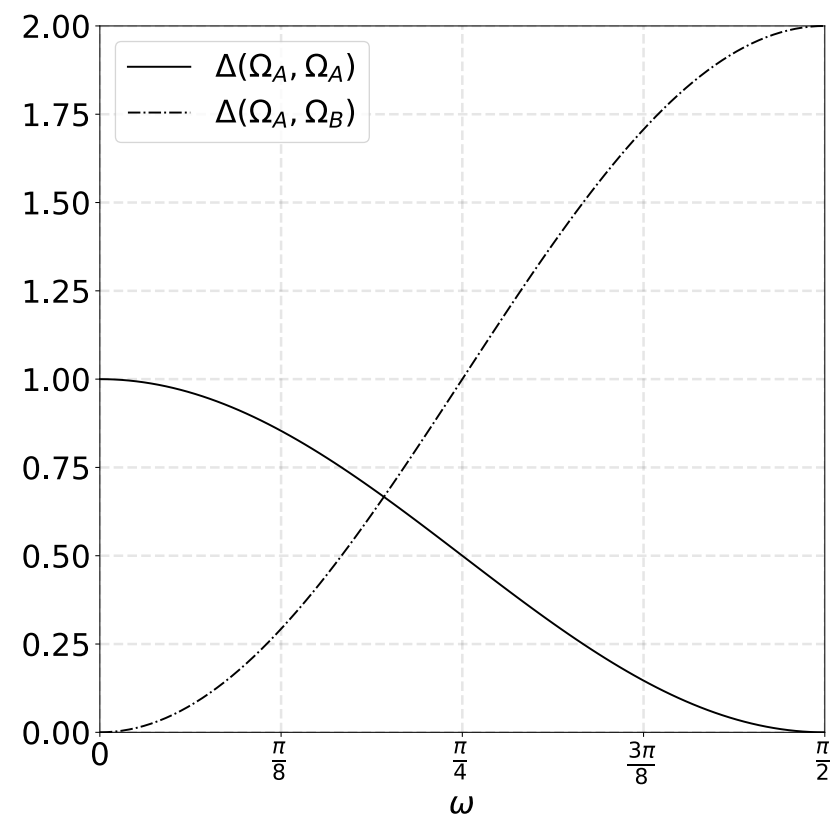

Fig. 1 Variation of the localization $\Delta\left(\Omega_{A}, \Omega_{A}\right)$ and delocalization index $\Delta\left(\Omega_{A}, \Omega_{B}\right)$ as a function of the parameter $\omega$.

of $\mathrm{H}_{2}(\omega=0)$, the delocalization index drops to zero, with a localization equal to one.

Although an increase in internuclear distance makes the bond weaker, in the Hartree-Fock wave function the relative importance of the covalent over the ionic valence bond structure remains the same. Still, a total decrease in bonding is not contradictory to keeping the same relative contributions. The situation is more problematic at equilibrium distance and upon the introduction of electron correlation. Including correlation increases the contribution of the covalent part of the valence bond type wave function (see equation (15)), which can be modeled by decreasing the value of $\omega$ (i.e. leading to larger values of $\cos (\omega))$. As observed in figure 1, this increases the localization index and decreases the delocalization index, which is again consistent with the findings of Wang et al. ${ }^{38}$ and the findings on aromatic systems in ground and excited states $^{39}$.

The key point is the paradox that from a valence bond perspective, an increase in the covalent contribution to the wave function, leads to a reduction in the delocalisation or shared electron distribution index. If we attach any value to "chemical intuition" it may appear discomforting that a higher weight of a covalent valence bond structure - usually considered the emblematic case of 
an electron sharing bond - results in a lower delocalisation index. According to Mayer ${ }^{3}$, this behavior of the delocalisation index upon inclusion of electron correlation is "not chemical" and these inadequacies render the delocalisation index unsuited for defining a bond order. On the other hand, according to Outeiral et al. ${ }^{33}$ this is not a "theoretical miscarriage" and the resulting delocalisation indices "offer a deeper understanding into the physical nature of bonding". Furthermore, Pendás and Francisco ${ }^{37}$ have recently pointed out that by abandoning the assumption that "covalency" can only be captured in terms of valence bond covalent structures the paradox in the behavior of the delocalisation index can also be resolved. This finding clearly shows that one should avoid relating the behavior of (de)localisation indices to the nature of the chemical bond being investigated without properly indicating what e.g. covalency is meant to reflect.

We suggest that interpretations where correlated methods are taken to disrupt electron sharing between the two domains ${ }^{7,32,33,40}$ be reexamined to add some more detail. At the very least one should carefully distinguish between chemical bonding and energy lowering. An observation that the energy lowering for $\mathrm{H}_{2}$ at equilibrium distance upon introduction of electron correlation goes hand in hand with a weakening of the chemical bond while it is of increasing covalent nature would most likely confuse many chemists. However this is a paradox; a lowered delocalisation index may reflect reduced "absolute" electron sharing although it may become at the same time relatively more covalent in the VB sense.

If we consider the chemical bond as the collection of all physical phenomena that result in energy lowering from two non-interacting hydrogen atoms to $\mathrm{H}_{2}$ at the full CI level, a bond index below one is then nothing unusual. Rather, from this viewpoint a bond index equal to one is an artefact of a too crude approximation to the exact wave function and should rather be considered as the discomforting number. Too much of chemical thinking on molecular electronic structures is based on essentially minimal basis Hartree-Fock theory where the "surprises" lie in the results obtained using properly correlating wave functions. Due to the current interest in strongly correlated and other interesting systems, any desired (new) chemical theory should be built on more appropriate wave functions. Notwithstanding the attractive interpretability of old theories, they should only be used very carefully.

The above reasoning relies on an analytical model with a rather peculiar set of atomic basis functions. However, this does not influence the conclusions. We performed full CI calculations for $\mathrm{H}_{2}$ using the STO-3G basis set. At the full CI equilibrium geometry we performed a Hartree-Fock calculation and projected the resulting Slater determinant on the valence bond structures (9) and (10) using the projection operators (13) and (14). As these wave functions are not orthogonal, the weights attached to both functions should take into account this non-orthogonality. The Hartree-Fock ground state function attaches equal (Mulliken) weights of $50 \%$ to both bond descriptions, covalent and ionic, as expected. When including electron correlation through the Full CI wave function at the equilibrium geometry, the covalent weight increases 
significantly, reaching $78.5 \%$. The delocalisation index, on the other hand, lowers from 1.00 at the Hartree-Fock level to 0.78 . So the total delocalisation index lowers but the relative importance of the covalent structure, the emblematic case of electron delocalisation, has increased. This is clearly not impossible as lowering in magnitude is independent of the relative magnitude of the contributions. Lengthening of the bond distance also reduces the delocalisation index although here again the covalent weight increases. For instance, increasing the distance by two ångström increases the covalent weight to $99.72 \%$ but at the same the delocalisation index lowers to virtually zero. In summary, if the delocalisation index can be considered as reflecting a degree of electron sharing in the wave function, the effect of electron correlation is to globally reduce electron sharing while at the same time increasing the covalent nature of the bond. This should be taken in to account to avoid possible misconceptions that reduced electron sharing would mean that the bond is less covalent.

\section{Conclusions}

Although (de)localization indices lead to chemically supposedly appealing results at the Hartree-Fock level of theory, introducing correlation reduces its appeal in terms of classical theoretical chemistry. In this study, we have shown using an analytical valence bond model that the decrease in electron sharing - as it would be testified by the delocalisation index - suggests that we think differently of the chemical bond. Indeed, in this analytic model it is precisely the larger contribution of the "electron sharing" covalent bond that leads to a smaller delocalization index and a larger localization index.

As such, the effect of electron correlation for $\mathrm{H}_{2}$ is best described as leading to a lowering in energy, that reduces the extent of electron sharing to the Hartree-Fock level of theory as testified by the delocalisation index, while at the same time increasing the relative importance of the covalent contribution to the total wave function.

Less complete statements might easily lead to misunderstandings or false assumptions. Still, most of the work using the delocalisation index relies on a number of assumptions or decisions in the definition of the latter, many of which are worth revisiting. We are currently investigating new indices properly linked to probabilities, that do not rely on self-pairing and make clearer distinctions between Fermi and Coulomb correlation and thus delay integration over e.g., spin to the latest phases. Such a probability based approach could constitute a third route to (de)localisation indices, next to density and (co)variance based approaches. We expect that the resulting indices will shed more light on the advantages and disadvantages of the indices discussed in this study. 


\section{References}

1. G. Frenking, S. Shaik, The chemical bond: fundamental aspects of chemical bonding (John Wiley \& Sons, 2014)

2. K. Ruedenberg, Reviews of Modern Physics 34(2), 326 (1962)

3. I. Mayer, Journal of computational chemistry 28(1), 204 (2007)

4. M. Giambiagi, M. Giambiagi, D.R. Grempel, C.D. Heymann, Journal de Chimie Physique 72, 15 (1975)

5. R.F. Bader, M.E. Stephens, Chemical Physics Letters 26(3), 445 (1974)

6. R.F. Bader, A. Streitwieser, A. Neuhaus, K.E. Laidig, P. Speers, Journal of the American Chemical Society 118(21), 4959 (1996)

7. X. Fradera, M.A. Austen, R.F. Bader, The Journal of Physical Chemistry A 103(2), 304 (1999)

8. R. Ponec, D.L. Cooper, Journal of Molecular Structure: THEOCHEM $\mathbf{7 2 7}(1-3), 133$ (2005)

9. K.B. Wiberg, Tetrahedron 24(3), 1083 (1968)

10. I. Mayer, Chemical Physics Letters 97(3), 270 (1983)

11. I. Mayer, Chemical Physics Letters 544, 83 (2012)

12. P. Bultinck, D.L. Cooper, R. Ponec, The Journal of Physical Chemistry A 114(33), 8754 (2010)

13. R. Ponec, Collection of Czechoslovak chemical communications 59(3), 505 (1994)

14. R. Ponec, A.J. Duben, Journal of computational chemistry 20(8), 760 (1999)

15. R. Ponec, J. Roithová, Theoretical Chemistry Accounts 105(4-5), 383 (2001)

16. R. Ponec, D.L. Cooper, Faraday discussions 135, 31 (2007)

17. M.S. de Giambiagi, M. Giambiagi, F.E. Jorge, Theoretica chimica acta 68(5), 337 (1985)

18. A.M. Pendás, E. Francisco, M. Blanco, Physical Chemistry Chemical Physics 9(9), 1087 (2007)

19. E. Francisco, A.M. Pendás, M. Blanco, The Journal of chemical physics 131(12), 124125 (2009)

20. R. Ponec, X. Gironés, The Journal of Physical Chemistry A 106(41), 9506 (2002)

21. R. Ponec, G. Yuzhakov, R. Carbó-Dorca, Journal of computational chemistry 24(15), 1829 (2003)

22. R. Ponec, G. Yuzhakov, M.R. Sundberg, Journal of computational chemistry 26(5), 447 (2005)

23. R. Ponec, D.L. Cooper, The Journal of Physical Chemistry A 111(44), 11294 (2007)

24. R. Ponec, G. Lendvay, J. Chaves, Journal of computational chemistry 29(9), 1387 (2008)

25. R. Ponec, F. Feixas, The Journal of Physical Chemistry A 113(29), 8394 (2009) 
26. R. Ponec, E. Ramos-Cordoba, P. Salvador, The Journal of Physical Chemistry A 117(9), 1975 (2013)

27. D.L. Cooper, R. Ponec, M. Kohout, Molecular Physics 114(7-8), 1270 (2016)

28. M. Giambiagi, M.S. de Giambiagi, K.C. Mundim, Structural Chemistry 1(5), 423 (1990)

29. P. Bultinck, R. Ponec, S. Van Damme, Journal of physical organic chemistry 18(8), $706(2005)$

30. F. Feixas, E. Matito, J. Poater, M. Solà, Journal of computational chemistry 29(10), 1543 (2008)

31. E. Francisco, A.M. Pendás, A. Costales, Physical Chemistry Chemical Physics 16(10), 4586 (2014)

32. J. Poater, M. Sola, M. Duran, X. Fradera, Theoretical Chemistry Accounts $\mathbf{1 0 7}(6), 362(2002)$

33. C. Outeiral, M.A. Vincent, A.M. Pendás, P.L. Popelier, Chemical Science (2018)

34. R. Ponec, D.L. Cooper, A. Savin, Chemistry-A European Journal 14(11), 3338 (2008)

35. W. Kutzelnigg, in Explicitly correlated wave functions in chemistry and physics: theory and applications, ed. by J. Rychlewski (Springer, 2003)

36. G. Frenking, A. Krapp, Journal of computational chemistry 28(1), 15 (2007)

37. A.M. Pendás, E. Francisco, Physical Chemistry Chemical Physics 20(18), $12368(2018)$

38. Y.G. Wang, C. Matta, N.H. Werstiuk, Journal of computational chemistry 24(14), 1720 (2003)

39. F. Feixas, J. Vandenbussche, P. Bultinck, E. Matito, M. Solà, Physical Chemistry Chemical Physics 13(46), 20690 (2011)

40. J. Poater, M. Duran, M. Solà, B. Silvi, Chemical reviews 105(10), 3911 (2005) 